\title{
ANALYSIS OF LINEAR AND NONLINEAR STIFF PROBLEMS USING THE RK-BUTCHER ALGORITHM
}

\author{
S. SEKAR
}

Received 25 July 2005; Accepted 10 November 2005

I present a numerical solution of linear and nonlinear stiff problems using the RK-Butcher algorithm. The obtained discrete solutions using the RK-Butcher algorithm are found to be very accurate and are compared with the exact solutions of the linear and nonlinear stiff problems and also with the Runge-Kutta method based on arithmetic mean (RKAM). A topic of stability for the RK-Butcher algorithm is discussed in detail. Error graphs for discrete and exact solutions are presented in a graphical form to show the efficiency of the RK-Butcher algorithm. The results obtained show that RK-Butcher algorithm is more useful for solving linear and nonlinear stiff problems and the solution can be obtained for any length of time.

Copyright (c) 2006 S. Sekar. This is an open access article distributed under the Creative Commons Attribution License, which permits unrestricted use, distribution, and reproduction in any medium, provided the original work is properly cited.

\section{Introduction}

Mathematical modeling has been used more and more in many areas such as in science, engineering, medicine, economics, and social sciences. Differential equations are one of the important and widely used techniques in mathematical modeling. However, not many differential equations have an analytic solution and even if there is one, usually it is extremely difficult to obtain and it is not very practical. Thus, numerical methods are truly a crucial part of solving differential equations which cannot be neglected. Since the late 18th century, numerical methods for solving differential equations have been developed continuously by many mathematicians. Later on in the 20th century, this subject made great improvements in the context of modern computers.

Among the models using differential equations (DEs), ordinary differential equations are frequently used to describe various physical problems, for example, motions of the planet in a gravity field like the Kepler problem, the simple pendulum, electrical circuits, and chemical kinetics problems. 
An ordinary differential equation (ODE) has the form

$$
y^{\prime}(x)=f(x, y(x))
$$

where $x$ is the independent variable which indicates the time in a physical problem and the dependent variable $y(x)$ is the solution. Moreover, since $y(x)$ could be an $N$-dimensional vector-valued function, the domain and range of the differential equation $f$ and the solution $y$ are given by

$$
\begin{aligned}
& f: \mathbb{R} \times \mathbb{R}^{N} \longrightarrow \mathbb{R}^{N}, \\
& y: \mathbb{R} \longrightarrow \mathbb{R}^{N} .
\end{aligned}
$$

The above equation (1.1) where $f$ is a function of both $x$ and $y$ is called "nonautonomous." However, by simply introducing an extra variable which is always exactly equal to $x$, it can be easily rewritten in an equivalent "autonomous" form below, where $f$ is a function of $y$ only:

$$
y^{\prime}(x)=f(y(x))
$$

Even though many problems are naturally expressed in the nonautonomous form, the autonomous form of differential equation (1.3) is preferred for most of the theoretical investigations. Furthermore, the autonomous form has some advantages in numerical analysis since it gives a greater possibility that numerical methods can solve the differential equation exactly.

The differential equation by itself is not enough to find a unique solution. Hence, some other additional information is needed. However, if all components of $y$ are given at a certain value of $x$, that is, "initial conditions," then the differential equation is called an "initial value problem (IVP)" which is closely and naturally involved with physical modeling.

An initial value problem with the given initial condition $y\left(x_{0}\right)=y_{0}$ has the structure

$$
y^{\prime}(x)=f(x, y(x)), \quad y\left(x_{0}\right)=y_{0},
$$

in nonautonomous form and

$$
y^{\prime}(x)=f(y(x)), \quad y\left(x_{0}\right)=y_{0},
$$

in autonomous form.

Runge-Kutta methods are being applied to determine numerical solutions for the problems which are modeled as initial value problems (IVPs) involving differential equations that arise in the fields of science and engineering by Alexander and Coyle [1], Murugesan et al. [5-9], Shampine [14], and Yaakub and Evans [15, 16]. Runge-Kutta methods have both advantages and disadvantages. Runge-Kutta methods are stable and easy to adapt for variable step size and order. However, they have difficulties in achieving high accuracy at reasonable cost, which was discussed recently by Butcher [2, 3]. 
Murugesan et al. [7, 8] have analyzed different second-order systems and multivariable linear systems via RK method based on centroidal mean. Park et al. [11, 12] have applied the RK-Butcher algorithm to optimal control of linear singular systems and observer design of singular systems (transistor circuits).

Murugesan et al. [10] and Sekar et al. [13] applied the RK-Butcher algorithm to industrial robot arm control problem and second-order IVPs. In this paper, I introduce the RK-Butcher algorithm for finding the numerical solution of linear and nonlinear stiff problems with more accuracy.

\section{RK-Butcher algorithm}

In [3], Butcher developed a new 6 stage order-5 RK method based on Taylor series to solve a first-order equation of the form (1.4).

Let $h$ denote the interval between equidistant values of $x$, then the fifth-order RKButcher algorithm formula of the $(n+1)$ th increment in $y$ is computed as

fifth-order predictor

$$
y_{n+1}=y_{n}+\frac{1}{90}\left(7 k_{1}+32 k_{3}+12 k_{4}+32 k_{5}+7 k_{6}\right) \text {; }
$$

fourth-order predictor

$$
y_{n+1}^{*}=y_{n}+\frac{1}{6}\left(k_{1}+4 k_{4}+k_{6}\right)
$$

local truncation error estimate (EE)

$$
\mathrm{EE}=y_{n+1}-y_{n+1}^{*},
$$

where

$$
\begin{aligned}
& k_{1}=h f\left(x_{n}, y_{n}\right), \\
& k_{2}=h f\left(x_{n}+\frac{h}{4}, y_{n}+\frac{k_{1}}{4}\right), \\
& k_{3}=h f\left(x_{n}+\frac{h}{4}, y_{n}+\frac{k_{1}}{8}+\frac{k_{2}}{8}\right), \\
& k_{4}=h f\left(x_{n}+\frac{h}{2}, y_{n}-\frac{k_{2}}{2}+k_{3}\right), \\
& k_{5}=h f\left(x_{n}+\frac{3 h}{4}, y_{n}+\frac{3 k_{1}}{16}+\frac{9 k_{4}}{16}\right), \\
& k_{6}=h f\left(x_{n}+h, y_{n}-\frac{3 k_{1}}{7}+\frac{2 k_{2}}{7}+\frac{12 k_{3}}{7}-\frac{12 k_{4}}{7}+\frac{8 k_{5}}{7}\right) .
\end{aligned}
$$


4 Stiff problems-RK-Butcher algorithm

\section{Stability}

The Runge-Kutta tableau given below is for the order-5 explicit method with 6 stages:

\begin{tabular}{c|cccccc}
0 & $\frac{1}{4}$ & $\frac{1}{4}$ & & & & \\
$\frac{1}{4}$ & $\frac{1}{8}$ & $\frac{1}{8}$ & & & & \\
$\frac{1}{2}$ & 0 & $-\frac{1}{2}$ & 1 & & & \\
$\frac{3}{4}$ & $\frac{3}{16}$ & 0 & 0 & $\frac{9}{16}$ & & \\
1 & $-\frac{3}{7}$ & $\frac{2}{7}$ & $\frac{12}{7}$ & $-\frac{12}{7}$ & $\frac{8}{7}$ & \\
\hline$\frac{7}{90}$ & 0 & $\frac{32}{90}$ & $\frac{12}{90}$ & $\frac{32}{90}$ & $\frac{7}{90}$ \\
& $\frac{1}{6}$ & 0 & 0 & $\frac{4}{6}$ & 0 & $\frac{1}{6}$
\end{tabular}

Consider the standard test problem $y^{\prime}=\lambda y$, where $\lambda$ is a constant and also complex in nature and it is used to determine the stability region of the method. The exact solution of this linear test problem $y^{\prime}=\lambda y$ is bounded, if $\operatorname{Re}(\lambda) \leq 0$. If the different order of RungeKutta method is applied to this test problem, then the "stability function" $R(z)$ is defined by

$$
\operatorname{Re}(z)=1+z b^{T}(I-z A)^{-1} 1 .
$$

However, to derive the stability function, let $z=h \lambda$. Then,

$$
\begin{aligned}
Y_{1} & =y_{0} \\
Y_{2} & =y_{0}+\frac{h}{4} F_{1}=\left(1+\frac{z}{4}\right) y_{0} \\
Y_{3} & =y_{0}+\frac{h}{8} F_{1}+\frac{h}{8} F_{2}=\left(1+\frac{z}{8}+\frac{z}{8}\left(1+\frac{z}{4}\right)\right) y_{0} \\
Y_{4} & =y_{0}-\frac{h}{2} F_{2}+h F_{3}=\left(1-\frac{z}{2}\left(1+\frac{z}{4}\right)+z\left(1+\frac{z}{8}+\frac{z}{8}\left(1+\frac{z}{4}\right)\right)\right) y_{0}, \\
Y_{5} & =y_{0}+\frac{3 h}{16} F_{1}+\frac{9 h}{16} F_{4} \\
& =\left(1+\frac{3 z}{16}\left(1+\frac{z}{4}\right)+\frac{9 z}{16}\left(1-\frac{z}{2}\left(1+\frac{z}{4}\right)+z\left(1+\frac{z}{8}+\frac{z}{8}\left(1+\frac{z}{4}\right)\right)\right)\right) y_{0},
\end{aligned}
$$




$$
\begin{aligned}
Y_{6}= & y_{0}-\frac{3 h}{7} F_{1}+\frac{2 h}{7} F_{2}+\frac{12 h}{7} F_{3}-\frac{12 h}{7} F_{4}+\frac{8 h}{7} F_{5} \\
= & \left(1-\frac{3 z}{7}+\frac{2 z}{7}\left(1+\frac{z}{4}\right)+\frac{12 z}{7}\left(1+\frac{z}{8}+\frac{z}{8}\left(1+\frac{z}{4}\right)\right)\right. \\
& -\frac{12 z}{7}\left(1-\frac{z}{2}\left(1+\frac{z}{4}\right)+z\left(1+\frac{z}{8}+\frac{z}{8}\left(1+\frac{z}{4}\right)\right)\right) \\
& \left.+\frac{8 z}{7}\left(1+\frac{3 z}{16}\left(1+\frac{z}{4}\right)+\frac{9 z}{16}\left(1-\frac{z}{2}\left(1+\frac{z}{4}\right)+z\left(1+\frac{z}{8}+\frac{z}{8}\left(1+\frac{z}{4}\right)\right)\right)\right)\right) y_{0},
\end{aligned}
$$

where

$$
\begin{aligned}
& F_{1}=\lambda y_{0}, \\
& F_{2}=\lambda\left(1+\frac{z}{4}\right) y_{0}, \\
& F_{3}=\lambda\left(1+\frac{z}{8}+\frac{z}{8}\left(1+\frac{z}{4}\right)\right) y_{0}, \\
& F_{4}=\lambda\left(1-\frac{z}{2}\left(1+\frac{z}{4}\right)+z\left(1+\frac{z}{8}+\frac{z}{8}\left(1+\frac{z}{4}\right)\right)\right) y_{0}, \\
& \begin{aligned}
Y_{5}=\lambda\left(1+\frac{3 z}{16}\left(1+\frac{z}{4}\right)+\frac{9 z}{16}\left(1-\frac{z}{2}\left(1+\frac{z}{4}\right)+z\left(1+\frac{z}{8}+\frac{z}{8}\left(1+\frac{z}{4}\right)\right)\right)\right) y_{0}, \\
Y_{6}=\lambda\left(1-\frac{3 z}{7}+\frac{2 z}{7}\left(1+\frac{z}{4}\right)+\frac{12 z}{7}\left(1+\frac{z}{8}+\frac{z}{8}\left(1+\frac{z}{4}\right)\right)\right. \\
\quad \quad-\frac{12 z}{7}\left(1-\frac{z}{2}\left(1+\frac{z}{4}\right)+z\left(1+\frac{z}{8}+\frac{z}{8}\left(1+\frac{z}{4}\right)\right)\right) \\
\left.\quad+\frac{8 z}{7}\left(1+\frac{3 z}{16}\left(1+\frac{z}{4}\right)+\frac{9 z}{16}\left(1-\frac{z}{2}\left(1+\frac{z}{4}\right)+z\left(1+\frac{z}{8}+\frac{z}{8}\left(1+\frac{z}{4}\right)\right)\right)\right)\right) y_{0} .
\end{aligned}
\end{aligned}
$$

Hence,

$$
\begin{aligned}
y_{1} & =y_{0}+\frac{h}{90}\left(7 F_{1}+32 F_{3}+12 F_{4}+32 F_{5}+7 F_{6}\right) \\
& =y_{0}\left(1+z+\frac{1}{2} z^{2}+\frac{1}{6} z^{3}+\frac{1}{24} z^{4}+\frac{1}{120} z^{5}+\frac{1}{720} z^{6}\right) .
\end{aligned}
$$

Note that $1+z+(1 / 2) z^{2}+(1 / 6) z^{3}+(1 / 24) z^{4}+(1 / 120) z^{5}+(1 / 720) z^{6}$ is the stability function, $R(z)$, which is the first 6 terms of $\exp (z)$ series. This illustrates that the stability function for an explicit Runge-Kutta method with 6 stages and order 5 is

$$
R(z)=1+z+\frac{z^{2}}{2 !}+\frac{z^{3}}{3 !}+\frac{z^{4}}{4 !}+\frac{z^{5}}{5 !}+\frac{z^{6}}{6 !}
$$




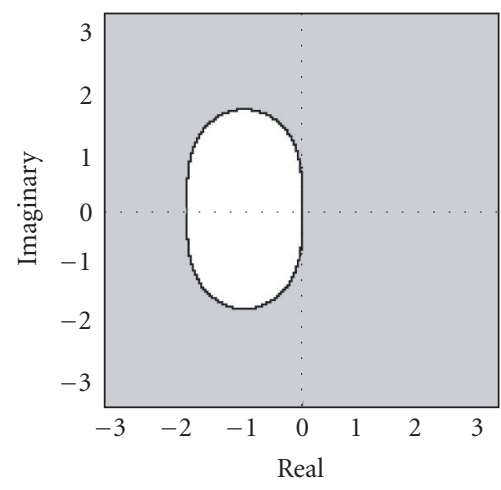

(a)

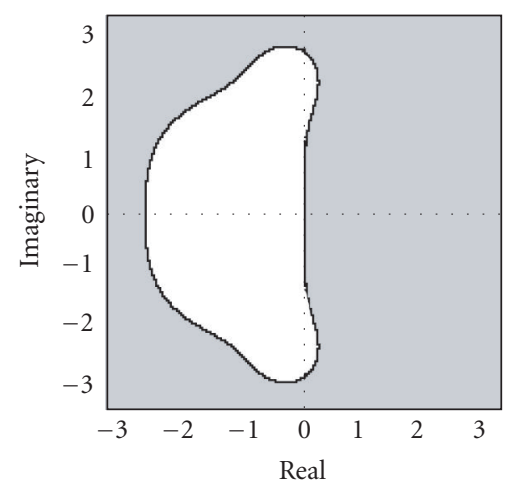

(c)

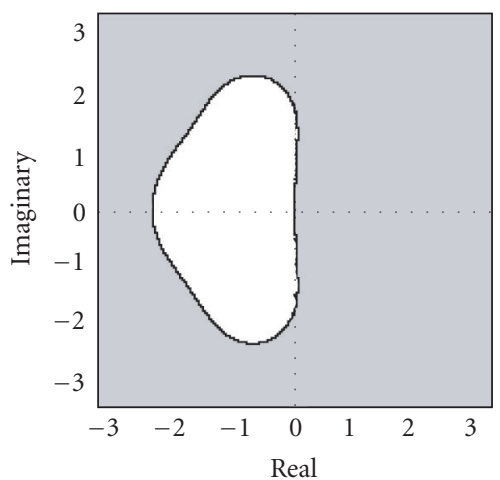

(b)

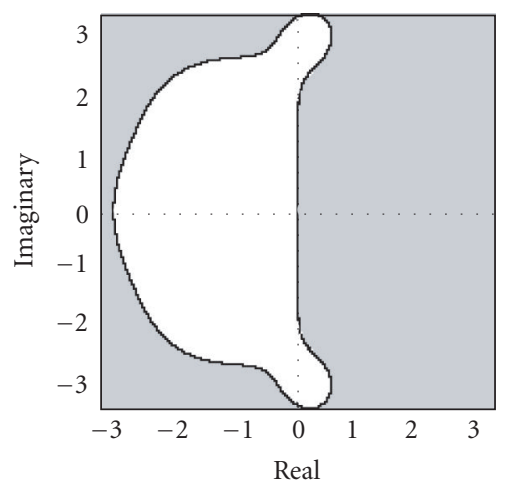

(d)

Figure 3.1. Stability regions for explicit Runge-Kutta methods of order 2 to 5 : (a) $p=2$; (b) $p=3$; (c) $p=4$; (d) $p=5$.

The plots of the boundaries of the stability regions defined by above functions are shown in Figure 3.1, where the unshaded part represents the stable region. In each case, the stability region is the bounded set enclosed by the curves. To find the boundaries, that is finding the values of $z$ for which $|R(z)=1|$, let $1+z+\left(z^{2} / 2 !\right)+\cdots+\left(z^{6} / 6 !\right)=\exp (i 2 \pi \theta)$ and find the roots of the polynomial. For example, when $p=6$,

$$
\begin{gathered}
1+z+\frac{z^{2}}{2 !}+\frac{z^{3}}{3 !}+\frac{z^{4}}{4 !}+\frac{z^{5}}{5 !}+\frac{z^{6}}{6 !}=e^{i 2 \pi \theta} \\
\left(1-e^{i 2 \pi \theta}\right)+z+\frac{z^{2}}{2}+\frac{z^{3}}{6}+\frac{z^{4}}{24}+\frac{z^{5}}{120}+\frac{z^{6}}{720}=0 .
\end{gathered}
$$

In Figure 3.1, $p=2$ and $p=3$ mentioned Runge-Kutta methods second and third orders, $p=4$ mentioned RKAM and $p=5$ mentioned RK-Butcher algorithm. 


\section{Stiff problems}

Even if there exists the numerical solution to a differential equation, certain types of differential equations are difficult to solve, in fact, they need certain types of numerical methods. This phenomenon known as "stiffness" was first recognized by Curtiss and Hirschfelder [4]. Stiffness occurs when some components of the solution decay much more rapidly than others. These problems have highly stable exact solutions but have highly unstable numerical solutions. There are several ways of characterizing "stiffness" and one way of understanding is looking at the Lipschitz constant. Stiff problems typically have a large Lipschitz constant; however, many of them have a more moderate size one-sided Lipschitz constant.

Definition 4.1 (Butcher [3]). The function $f:[a, b] \times \mathbb{R}^{N} \rightarrow \mathbb{R}^{N}$ is said to satisfy a "onesided Lipschitz condition" if there exists a "one-sided Lipschitz constant" $l$, such that for all $x \in[a, b]$ and all $y, z \in \mathbb{R}^{N}$,

$$
\langle f(x, y)-f(x, z), y-z\rangle \leq l\|y-z\|^{2},
$$

where the norm is defined by $\|y\|^{2}=\langle y, y\rangle$ assuming that there exists an inner product on $\mathbb{R}^{N}$.

Therefore, the Lipschitz constant could be large while the one-sided Lipschitz constant could be small, or even negative. The following theorem leads me to deduce the following result.

Theorem 4.2 (Butcher [3]). If $f$ satisfies a one-sided Lipschitz condition with one-sided Lipschitz constant $l$, and $y$ and $z$ are solutions of $y^{\prime}(x)=f(x, y(x))$, then for all $x \geq x_{0}$,

$$
\|y(x)-z(x)\| \leq \exp \left(l\left(x-x_{0}\right)\right)\left\|y\left(x_{0}\right)-z\left(x_{0}\right)\right\|
$$

Notice from this result that the distance between any two solutions will not increase rapidly or may even decrease if the equation has an adequate one-sided Lipschitz constant. Since stiffness is closely related to the behaviour of perturbations to a given solution, it is important to find out the effect of small perturbations with a one-sided Lipschitz condition.

Consider

$$
y^{\prime}(x)=f(x, y(x))
$$

with $y(x)$, a solution, and $\varepsilon Y(x)$, a small perturbation to the given solution. Replace $y(x)$ in (4.3) by $y(x)+\varepsilon Y(x)$ and expand the solution in a series in powers of $\varepsilon$ up to the second 
order, then get

$$
y^{\prime}(x)+\varepsilon Y^{\prime}(x)=f(x, y(x))+\varepsilon \frac{\partial f}{\partial y} Y(x)
$$

Subtract (4.3) from (4.4) and simplify it, then finally obtain the equation which controls the behaviour of the perturbation,

$$
\begin{aligned}
Y^{\prime}(x) & =\frac{\partial f}{\partial y} Y(x) \\
& =J(x) Y(x),
\end{aligned}
$$

where $J(x)$ is the Jacobian matrix of $f(x, y(x))$. I can use the spectrum of eigenvalues of $J(x)$ to characterize stiffness. The eigenvalues of $J(x)$ determine the growth rate of the perturbation with a moderate change in the value of the solution and a very small change in $J(x)$ in a time interval $\Delta x$. The existence of one or more large and negative values of $\lambda$, where $\lambda \in \sigma(J(x))$ and where $x \in \Delta x$ indicates that stiffness is present.

\section{Examples of stiff problem}

Stiffness can be understood by the practical difficulty found in numerical calculation as well. The stiff problems are impossible or very difficult to solve by explicit methods, mainly because the small bounded stability region of explicit methods forces the numerical method to take very small step sizes for the smooth solution. Two examples of stiff problems are given here to observe how explicit and implicit methods work for these problems.

Example 5.1 (stiff linear problem). Consider the stiff system of three linear ordinary differential equations with corresponding initial conditions of the form (1.4),

$$
\left[\begin{array}{l}
y_{1}^{\prime}(x) \\
y_{2}^{\prime}(x) \\
y_{3}^{\prime}(x)
\end{array}\right]=\left[\begin{array}{ccc}
0 & 1 & 0 \\
-1 & 0 & 0 \\
-L & 1 & L
\end{array}\right]\left[\begin{array}{l}
y_{1}(x) \\
y_{2}(x) \\
y_{3}(x)
\end{array}\right], \quad\left[\begin{array}{l}
y_{1}(0) \\
y_{2}(0) \\
y_{3}(0)
\end{array}\right]=\left[\begin{array}{l}
0 \\
1 \\
\varepsilon
\end{array}\right],
$$

where $L=-25$ and $\varepsilon=2$.

The analytic solution is

$$
\left[\begin{array}{l}
y_{1}(x) \\
y_{2}(x) \\
y_{3}(x)
\end{array}\right]=\left[\begin{array}{c}
\sin (x) \\
\cos (x) \\
\sin (x)+\varepsilon \exp (L x)
\end{array}\right]
$$

which is drawn in Figure 5.1. 


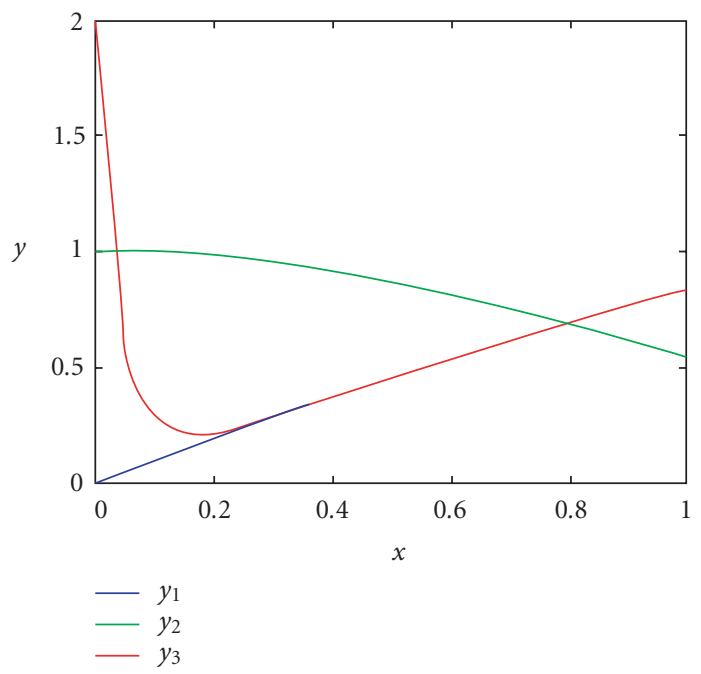

Figure 5.1. Analytical solution of Example 5.1, stiff linear problem, exact solution on $[0,1]$.

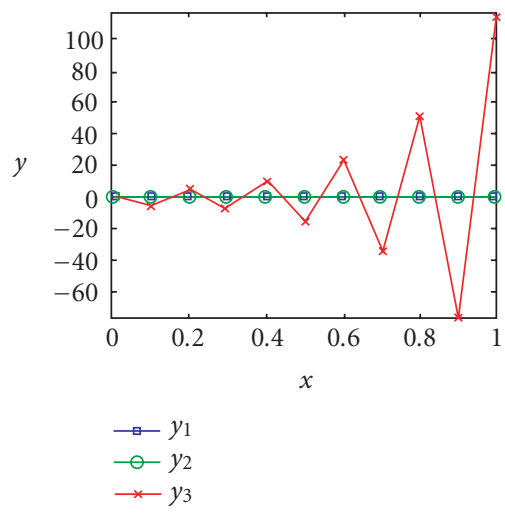

(a)

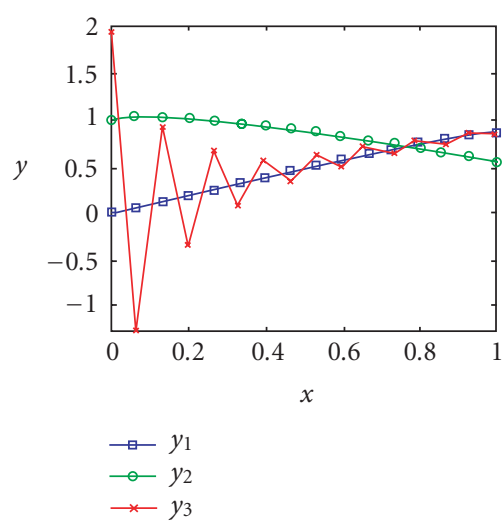

(b)

Figure 5.2. RKAM solution of Example 5.1 with $n=4$ and 8.

The results of using the RKAM and RK-Butcher algorithm methods for solving this stiff problem on the interval of $[0,1]$ are presented in Figures 5.2, 5.3, 5.4, 5.5, 5.6, and 5.7. Figures 5.2-5.4 show that the RKAM method definitely seems to have difficulty approximating $y_{3}$ while $y_{1}$ and $y_{2}$ are computed without difficulties. Especially, the approximations with $n=10$ and $n=15$ are hopeless. However, the RK-Butcher algorithm method performs perfectly well even for $n$ as low as 4 as shown in Figures 5.5-5.7. 


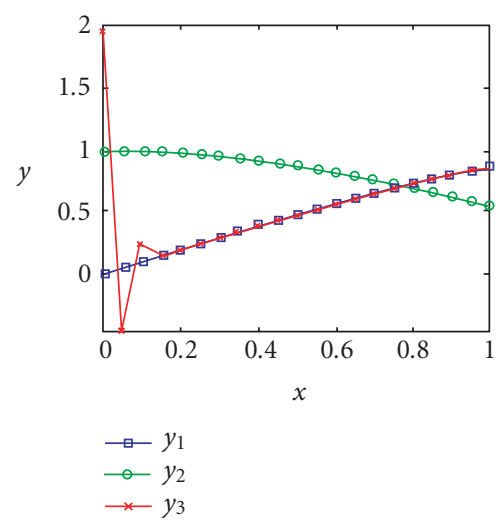

(a)

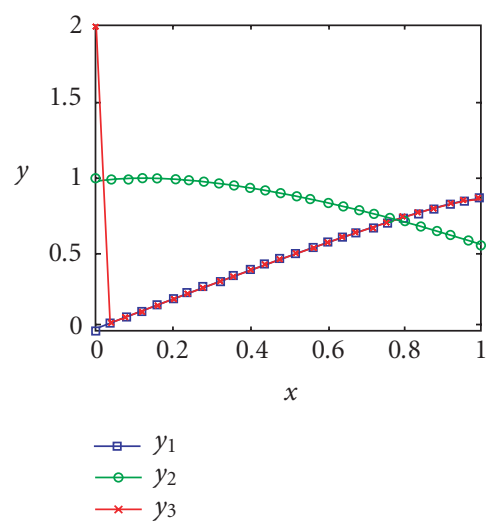

(b)

Figure 5.3. RKAM solution of Example 5.1 with $n=12$ and 16 .

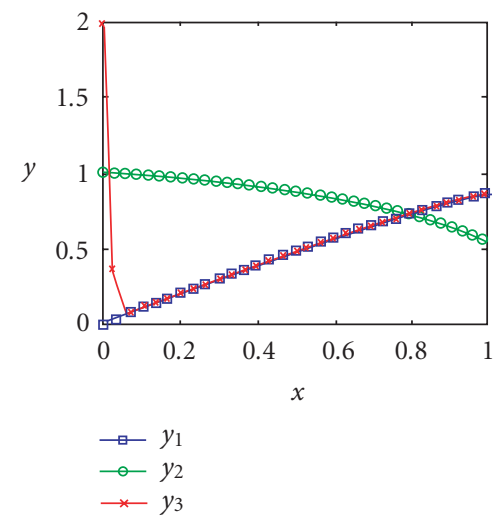

(a)

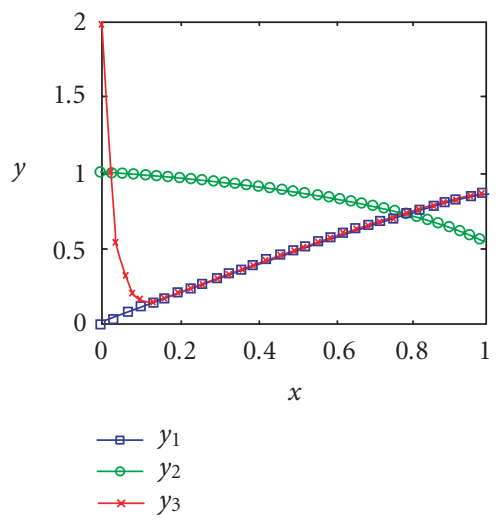

(b)

Figure 5.4. RKAM solution of Example 5.1 with $n=20$ and 30 .

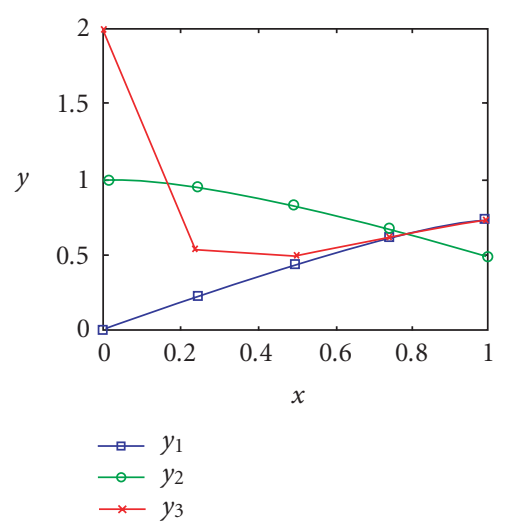

(a)

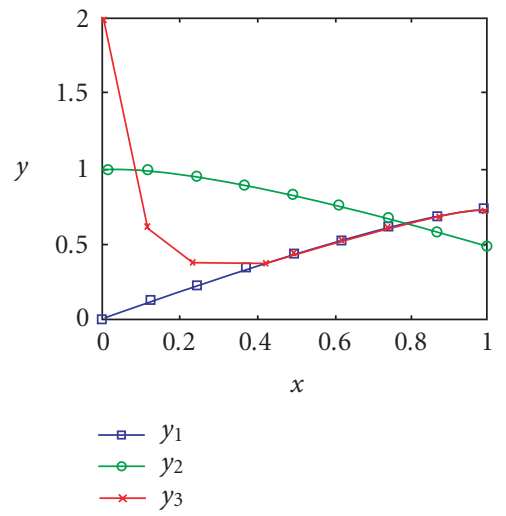

(b)

Figure 5.5. RK-Butcher algorithm solution of Example 5.1 with $n=4$ and 8 . 


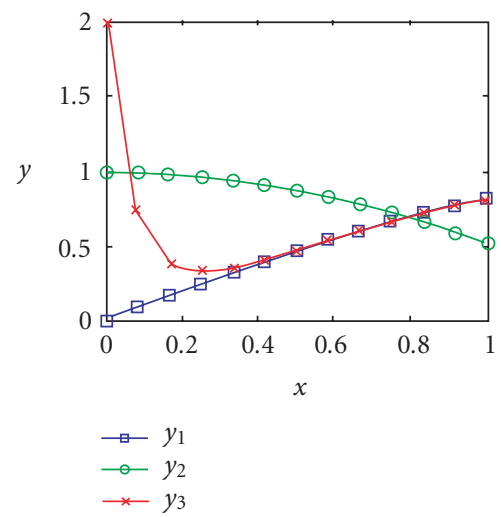

(a)

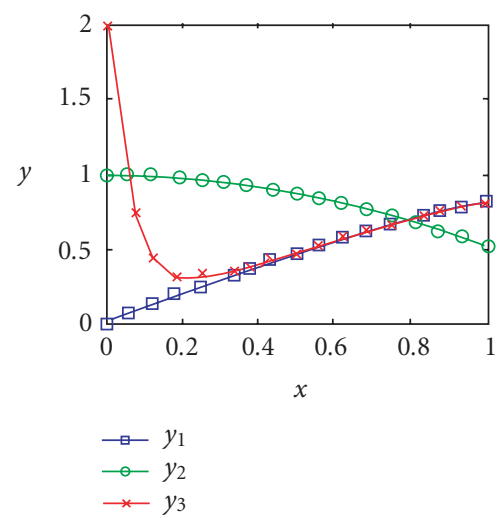

(b)

Figure 5.6. RK-Butcher algorithm solution of Example 5.1 with $n=12$ and 16.

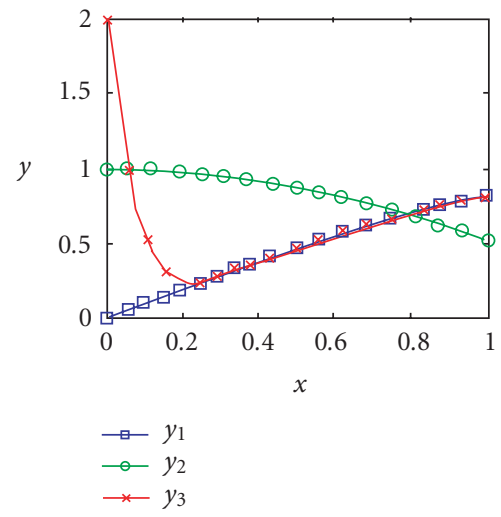

(a)

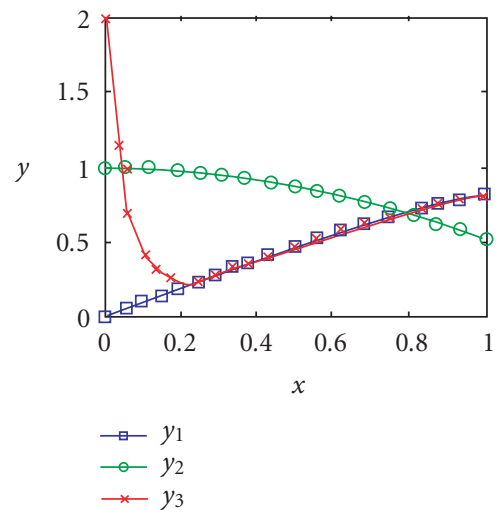

(b)

Figure 5.7. RK-Butcher algorithm solution of Example 5.1 with $n=20$ and 30.

Example 5.2 (stiff nonlinear problem (the Kaps problem)). Consider the stiff system of two-dimensional Kaps problem with corresponding initial conditions of the form (1.4),

$$
\left[\begin{array}{l}
y_{1}^{\prime}(x) \\
y_{2}^{\prime}(x)
\end{array}\right]=\left[\begin{array}{c}
-1002 y_{1}(x)+1000 y_{2}(x)^{2} \\
y_{1}(x)-y_{2}(x)\left(1+y_{2}(x)\right)
\end{array}\right], \quad\left[\begin{array}{l}
y_{1}(0) \\
y_{2}(0)
\end{array}\right]=\left[\begin{array}{l}
1 \\
1
\end{array}\right] \text {. }
$$

Analytic solution is

$$
\left[\begin{array}{l}
y_{1}(x) \\
y_{2}(x)
\end{array}\right]=\left[\begin{array}{c}
\exp (-2 x) \\
\exp (-x)
\end{array}\right]
$$

which is drawn in Figure 5.8 . 


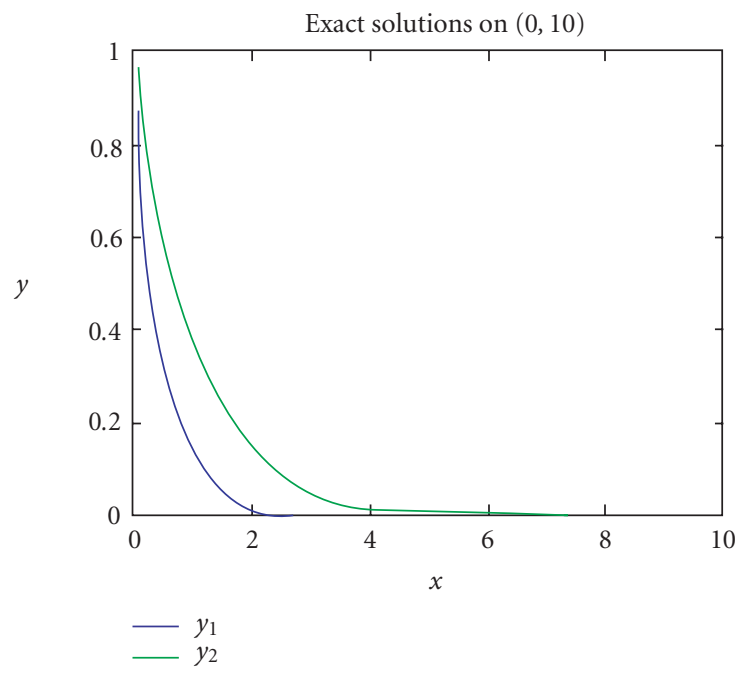

Figure 5.8. Analytical solution of Example 5.2, stiff nonlinear problem, exact solution on $[0,10]$.

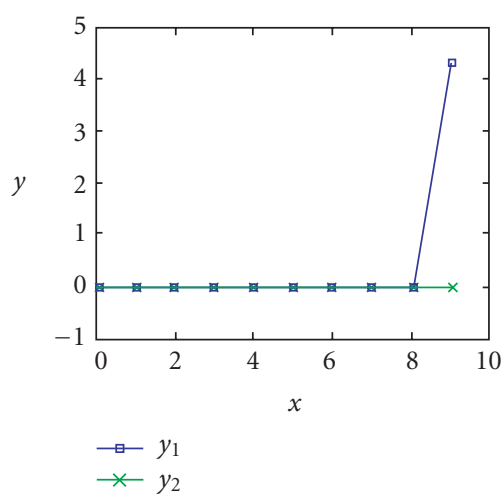

(a)

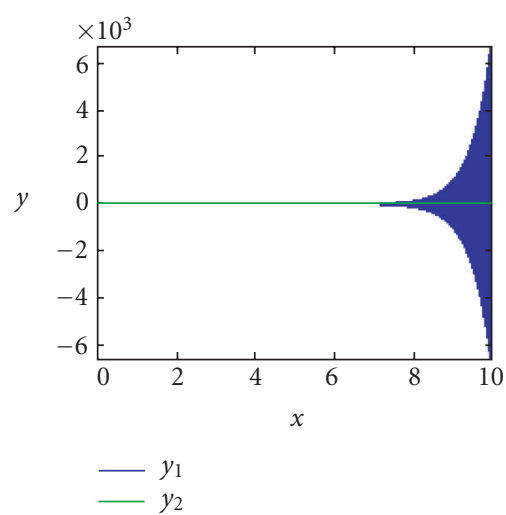

(b)

Figure 5.9. RKAM solution of Example 5.2 with $n=4$ and 8 .

In Figures 5.9, 5.10, 5.11, 5.12, 5.13, and 5.14, the computed solutions of this problem using the RKAM and RK-Butcher algorithm method on the interval of $[0,10]$ are displayed. Even using a large number of steps, the RKAM method performs poorly. However the RK-Butcher algorithm method easily gives a good approximation.

From these two examples, it is clearly confirmed that the RKAM method is not suitable but the RK-Butcher algorithm method is appropriate for stiff problems. 


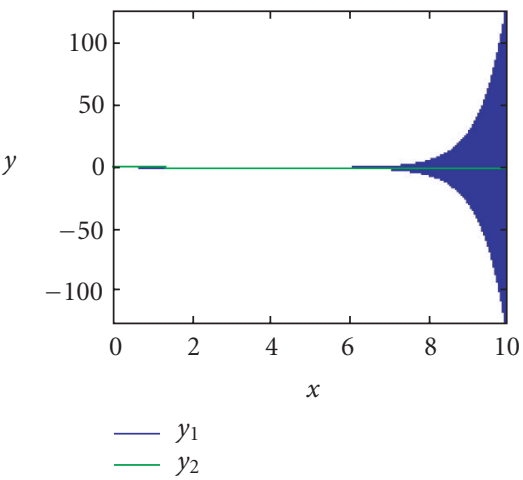

(a)

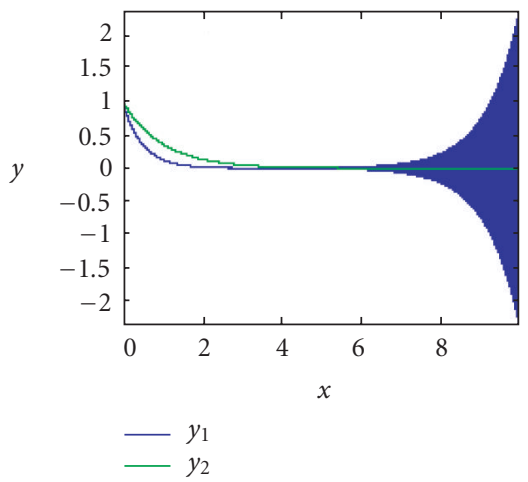

(b)

Figure 5.10. RKAM solution of Example 5.2 with $n=12$ and 16 .

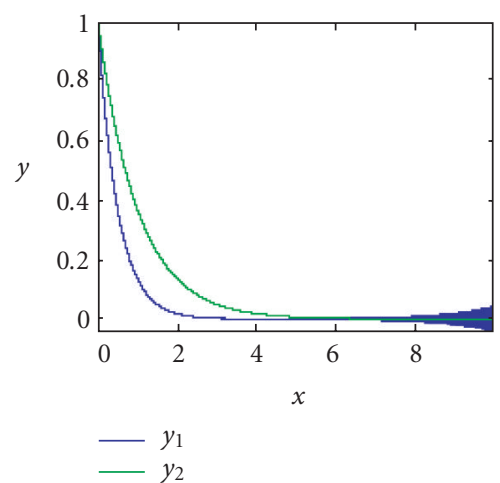

(a)

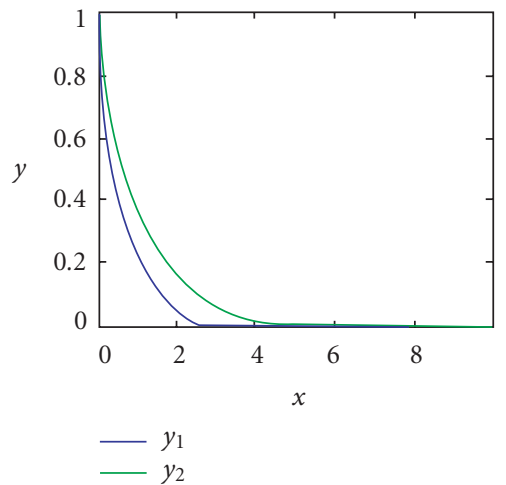

(b)

Figure 5.11. RKAM solution of Example 5.2 with $n=20$ and 30 .

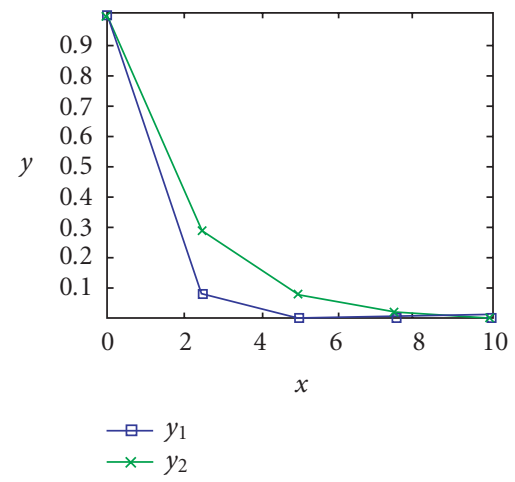

(a)

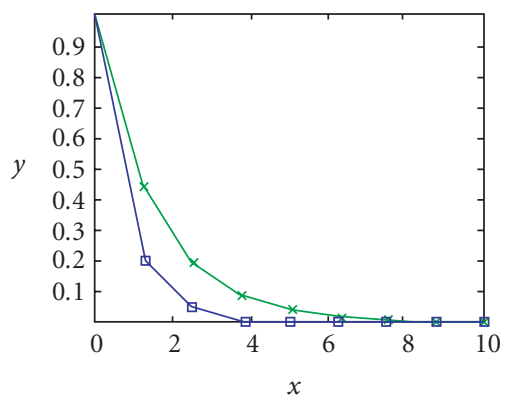

$\square y_{1}$

$-y_{2}$

Figure 5.12. RK-Butcher algorithm solution of Example 5.2 with $n=4$ and 8 . 


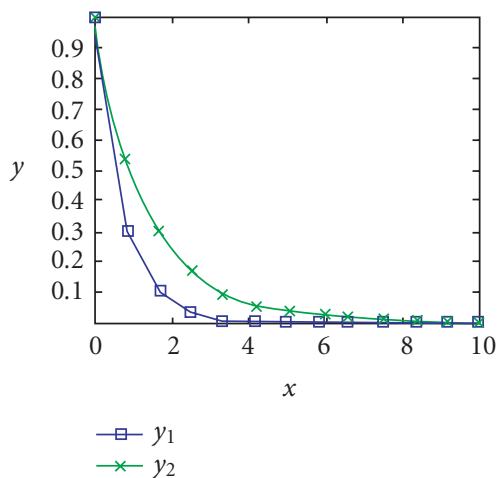

(a)

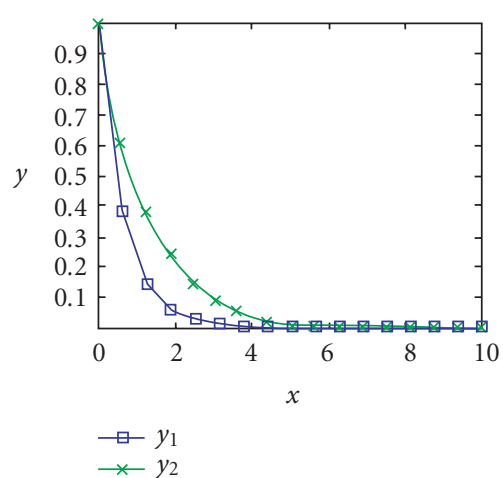

(b)

Figure 5.13. RK-Butcher algorithm solution of Example 5.2 with $n=12$ and 16 .

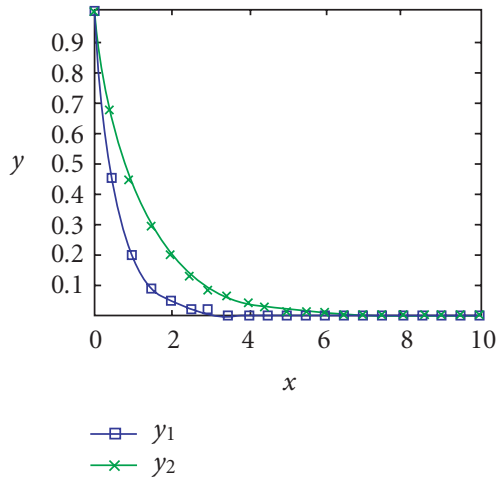

(a)

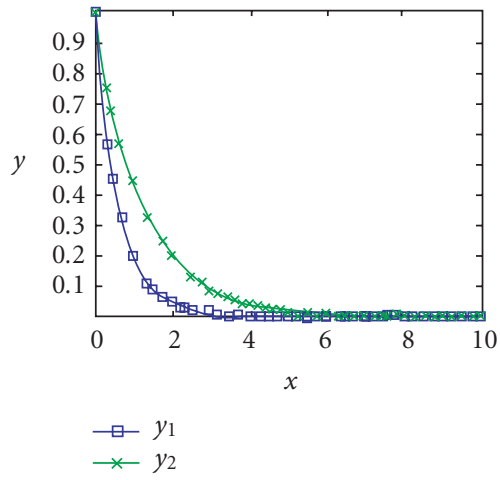

(b)

Figure 5.14. RK-Butcher algorithm solution of Example 5.2 with $n=20$ and 30 .

\section{Conclusions}

The RKAM method is simple. It uses only four pieces of information from the past and evaluates the driving function only four per step. However, the RKAM method is not very practical for computational purpose since considerable computational effort is required to improve accuracy. The study of stability of the RK-Butcher algorithm reveals that it (RK-Butcher algorithm) converges faster than the other standard ordinary differential equation solvers (see Butcher [3]). The stability regions are given in Figure 3.1. From Figures 5.2-5.7 and Figures 5.9-5.14, one can predict that the error is less in RK-Butcher algorithm when compared to the RKAM method. This RK-Butcher algorithm provided a momentum for advancing numerical methods for solving linear and nonlinear stiff problems. 


\section{References}

[1] R. K. Alexander and J. J. Coyle, Runge-Kutta methods and differential-algebraic systems, SIAM Journal on Numerical Analysis 27 (1990), no. 3, 736-752.

[2] J. C. Butcher, The Numerical Analysis of Ordinary Differential Equations. Runge-Kutta and General Linear Methods, A Wiley-Interscience Publication, John Wiley \& Sons, Chichester, 1987.

[3] _ Numerical Methods for Ordinary Differential Equations, John Wiley \& Sons, Chichester, 2003.

[4] C. F. Curtiss and J. O. Hirschfelder, Integration of stiff equations, Proceedings of the National Academy of Sciences of the United States of America 38 (1952), 235-243.

[5] K. Murugesan, D. P. Dhayabaran, E. C. H. Amirtharaj, and D. J. Evans, A comparison of extended Runge-Kutta formulae based on variety of means to solve system of IVPs, International Journal of Computer Mathematics 78 (2001), no. 2, 225-252.

[6] __ A fourth order embedded Runge-Kutta RKACeM $(4,4)$ method based on arithmetic and centroidal means with error control, International Journal of Computer Mathematics 79 (2002), no. 2, 247-269.

[7] K. Murugesan, D. P. Dhayabaran, and D. J. Evans, Analysis of different second order systems via Runge-Kutta method, International Journal of Computer Mathematics 70 (1999), no. 3, 477493.

[8] __ Analysis of second order multivariable linear system using single term Walsh series technique and Runge-Kutta method, International Journal of Computer Mathematics 72 (1999), no. 3, 367-374.

[9] __ Analysis of non-linear singular system from fluid dynamics using extended Runge-Kutta methods, International Journal of Computer Mathematics 76 (2000), no. 2, 239-266.

[10] K. Murugesan, S. Sekar, V. Murugesh, and J. Y. Park, Numerical solution of an industrial robot arm control problem using the RK-Butcher algorithm, International Journal of Computer Applications in Technology 19 (2004), no. 2, 132-138.

[11] J. Y. Park, D. J. Evans, K. Murugesan, S. Sekar, and V. Murugesh, Optimal control of singular systems using the RK-Butcher algorithm, International Journal of Computer Mathematics 81 (2004), no. 2, 239-249.

[12] J. Y. Park, K. Murugesan, D. J. Evans, S. Sekar, and V. Murugesh, Observer design of singular systems (transistor circuits) using the RK-Butcher algorithms, International Journal of Computer Mathematics 82 (2005), no. 1, 111-123.

[13] S. Sekar, V. Murugesh, and K. Murugesan, Numerical strategies for the system of second order IVPS using the RK-Butcher algorithms, International Journal of Computer Science and Applications 1 (2004), no. 2, 96-117.

[14] L. F. Shampine, Numerical Solution of Ordinary Differential Equations, Chapman \& Hall, New York, 1994.

[15] A. R. Yaakub and D. J. Evans, A fourth order Runge-Kutta RK $(4,4)$ method with error control, International Journal of Computer Mathematics 71 (1999), no. 3, 383-411.

[16] _ New Runge-Kutta starters for multistep methods, International Journal of Computer Mathematics 71 (1999), no. 1, 99-104.

S. Sekar: Department of Mathematics, National Institute of Technology, Tiruchirappalli, Tanjore Road, Tiruchirappalli - 620 015, Tamil Nadu, India

E-mail address: ssekar_psg@yahoo.com 


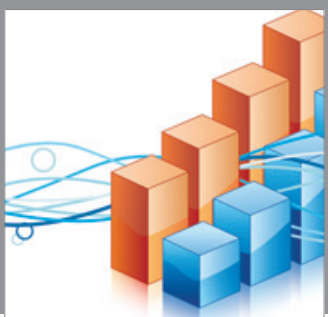

Advances in

Operations Research

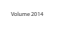

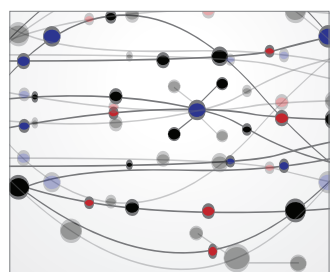

\section{The Scientific} World Journal
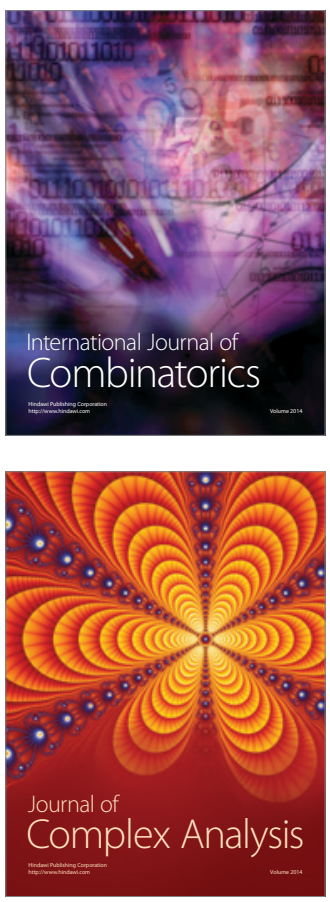

International Journal of

Mathematics and

Mathematical

Sciences
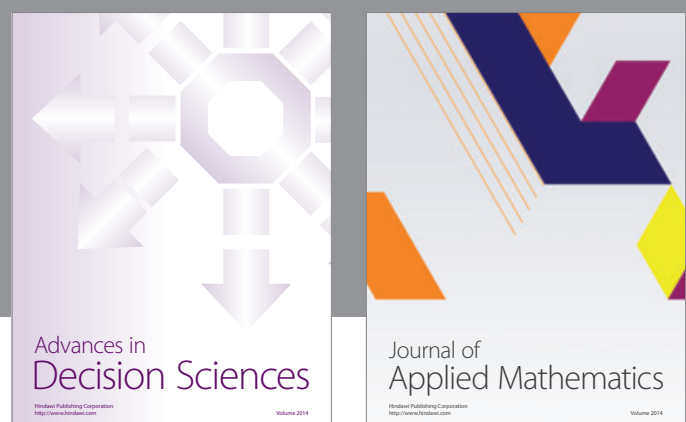

Journal of

Applied Mathematics
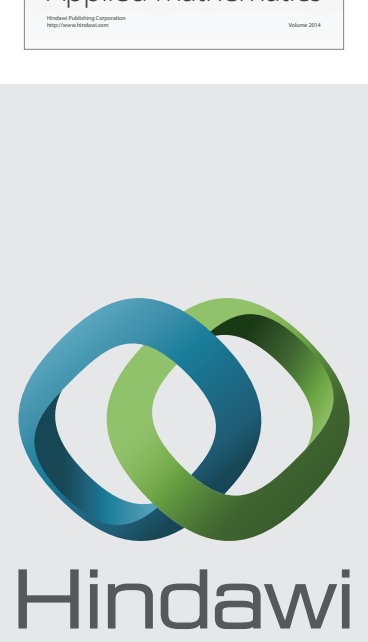

Submit your manuscripts at http://www.hindawi.com
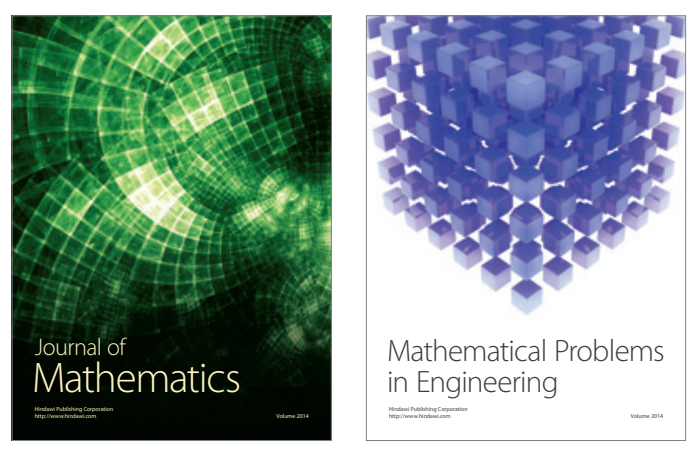

Mathematical Problems in Engineering
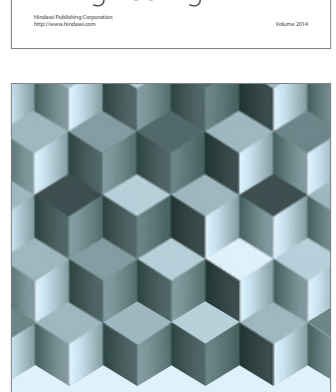

Journal of

Function Spaces
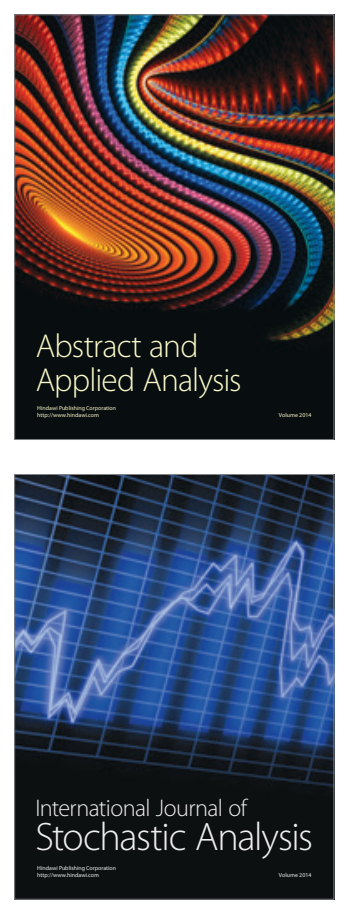

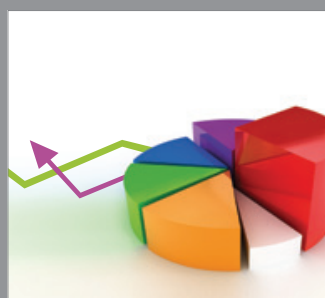

ournal of

Probability and Statistics

Promensencen
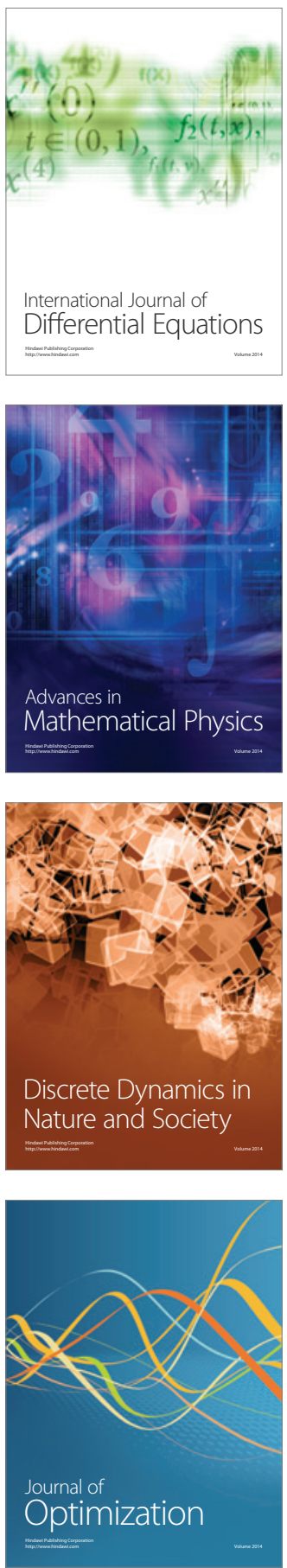